\title{
The role of SMAD3 in the genetic predisposition to papillary thyroid carcinoma
}

\author{
Yanqiang Wang, PhD ${ }^{1}$, Huiling He, MD ${ }^{1}$, Sandya Liyanarachchi, MSc, MAS ${ }^{1}$, Luke K. Genutis, BS ${ }^{1}$, \\ Wei Li, MD ${ }^{1}$, Lianbo Yu, PhD ${ }^{2,3}$, John E. Phay, MD ${ }^{4}$, Rulong Shen, MD ${ }^{5}$, Pamela Brock, MS, $\mathrm{LGC}^{6}$ and \\ Albert de la Chapelle, MD, $\mathrm{PhD}^{1}$
}

Purpose: To identify and characterize the functional variants, regulatory gene networks, and potential binding targets of SMAD3 in the $15 \mathrm{q} 22$ thyroid cancer risk locus.

Methods: We performed linkage disequilibrium (LD) and haplotype analyses to fine map the 15q22 locus. Luciferase reporter assays were applied to evaluate the regulatory effects of the candidate variants. Knockdown by small interfering RNA, microarray analysis, chromatin immunoprecipitation (ChIP) and quantitative real-time polymerase chain reaction assays were performed to reveal the regulatory gene network and identify its binding targets.

Results: We report a 25.6-kb haplotype within SMAD3 containing numerous single-nucleotide polymorphisms (SNPs) in high LD. SNPs rs17293632 and rs4562997 were identified as functional variants of $S M A D 3$ by luciferase assays within the $\mathrm{LD}$ region. These variants regulate SMAD3 transcription in an allele-specific manner through enhancer elements in introns of SMAD3. Knockdown of $S M A D 3$ in thyroid cancer cell lines revealed its regulatory gene network including two upregulated genes, SPRY4 and SPRY4-IT1. Sequence analysis and ChIP assays validated the actual binding of SMAD3 protein to multiple SMAD binding element sites in the region upstream of SPRY4.

Conclusion: Our data provide a functional annotation of the 15 q22 thyroid cancer risk locus.

Genet Med advance online publication 4 January 2018

Key Words: $15 \mathrm{q} 22$ locus; downstream targets; functional variants; papillary thyroid carcinoma; SMAD3

\section{INTRODUCTION}

Thyroid cancer is the most common endocrine malignancy. According to the U.S. National Cancer Institute, the estimated number of new thyroid cancers in 2017 is 56,870, with an estimated 2,010 deaths (http://seer.cancer.gov/statfacts/html/ thyro.html). The majority of thyroid cancers are differentiated thyroid cancers, including papillary and follicular thyroid carcinomas. Papillary thyroid carcinoma (PTC) accounts for $75-85 \%$ of all thyroid cancer.

It is well known that PTC displays strong heritability; however, the predisposition to PTC is complex and most likely due to multiple mutations. ${ }^{1}$ The driver mutations can confer distinct gene expression patterns, signaling, and clinical characteristics of PTC. ${ }^{2}$ In the past few years, a series of thyroid cancer risk loci have been identified by genome-wide association studies (GWAS). ${ }^{3}$ Functional aspects of the risk alleles in some of these loci have been extensively explored. ${ }^{4,5}$ Recently, another GWAS revealed five novel loci that were found to be significantly correlated with thyroid cancer risk. ${ }^{6}$ Of these, two variants at the $15 \mathrm{q} 22$ locus showed independent association with thyroid cancer $(\mathrm{rs} 2289261[\mathrm{C}]$ with odds ratio $(\mathrm{OR})=1.23$;
$P=3.1 \times 10^{-9}$, and $\mathrm{rs56062135}[\mathrm{T}]$ with $\mathrm{OR}=1.24$; $\left.P=4.9 \times 10^{-9}\right)$. These two single-nucleotide polymorphisms (SNPs) are located in separate introns of the SMAD family member 3 gene (SMAD3), however, functional roles for these variants have not been demonstrated. The putative molecular mechanisms leading to thyroid cancer susceptibility are yet undefined.

SMAD3 has been shown to be involved in the induction of apoptosis, metastasis, and tumor progression. ${ }^{7-9}$ As a key signaling molecule of the transforming growth factor- $\beta$ (TGF$\beta$ ) pathway, loss of $S M A D 3$ expression can increase cancer susceptibility in gastric cancer and T-cell acute lymphoblastic leukemia. ${ }^{10,11}$ Previously, some missense mutations of SMAD3 were reported to reduce its effect on TGF- $\beta$-induced transcriptional activation in certain cancer types. ${ }^{12,13}$ SMAD3 protein interacts with other DNA-binding cofactors to either activate or repress the transcription of specific target genes. ${ }^{14,15}$ SMAD3 functions in a cell type-specific manner to regulate genes in TGF- $\beta$ signaling. While a number of SMAD3 downstream targets have been discovered in cell models, ${ }^{16,17}$ little is known regarding such targets in thyroid cancer.

\footnotetext{
${ }^{1}$ Human Cancer Genetics Program and Department of Cancer Biology and Genetics, The Ohio State University Comprehensive Cancer Center, The Ohio State University, Columbus, Ohio, USA; ${ }^{2}$ Center for Biostatistics, The Ohio State University, Columbus, Ohio, USA; ${ }^{3}$ Department of Biomedical Informatics, The Ohio State University, Columbus, Ohio, USA; ${ }^{4}$ Department of Surgery, The Ohio State University Comprehensive Cancer Center, The Ohio State University, Columbus, Ohio, USA; ${ }^{5}$ Department of Pathology, The Ohio State University Comprehensive Cancer Center, The Ohio State University, Columbus, Ohio, USA; ${ }^{6}$ Department of Internal Medicine, The Ohio State University Comprehensive Cancer Center, The Ohio State University, Columbus, Ohio, USA. Correspondence: Albert de la Chapelle (albert.delachapelle@osumc.edu) 


\section{ORIGINAL RESEARCH ARTICLE}

SMAD3 shows higher expression in thyroid tissue compared with most other tissues, ${ }^{6}$ suggesting a potential role in the predisposition to thyroid cancer and the maintenance of normal thyroid function. This hypothesis is supported by the fact that the thyroid cancer risk alleles of the two GWAS variants are associated with decreased expression of SMAD3 in normal thyroid tissue in the GTEx database (http://www. gtexportal.org/home/). The purpose of our study was to identify the functional variants of SMAD3 in the $15 \mathrm{q} 22$ thyroid cancer risk locus, and to investigate the mechanisms by which alterations of SMAD3 contribute to thyroid cancer susceptibility.

\section{MATERIALS AND METHODS}

The study was approved by the Institutional Review Board at the Ohio State University, and all subjects gave written informed consent before participation.

\section{Thyroid cancer cell lines and cell culture}

The TPC-1 and BCPAP cell lines were incubated in antibiotic-free DMEM or RPMI 1640 medium, respectively, supplemented with $10 \%$ (vol/vol) fetal bovine serum (Thermo Fisher Scientific, Waltham, MA) at $37^{\circ} \mathrm{C}$ in humidified air with $5 \%(\mathrm{vol} / \mathrm{vol}) \mathrm{CO}_{2}$. The cell lines were obtained from Rebecca Schweppe (University of Colorado Cancer Center, Denver, CO). We reauthenticate each cell line by DNA fingerprinting upon receipt. Frozen stocks are reanalyzed periodically every $2-3$ years.

\section{Linkage disequilibrium and haplotype analyses}

Linkage disequilibrium (LD) analysis of the SMAD3 gene was performed using the genotype data from 1000 Genomes Project (phase 3), European population of 503 samples. Haploview V4.2 software was applied and haplotype blocks were generated using the confidence interval method. ${ }^{18}$ Haplotypes of the six selected SNPs were generated by using the genotyping and computer imputation data from the European descendants in an Ohio cohort of the recent GWAS. ${ }^{6}$ The SHAPEIT V2 program $^{19}$ was used to estimate the haplotype frequencies in 1,359 PTC cases and 1,605 controls. $P$ values and OR are provided using Fisher's exact test to compare each haplotype with the rest of the haplotypes.

\section{Generation of plasmid constructs}

The six putative regulatory regions containing the corresponding SNPs in SMAD3 introns were polymerase chain reaction (PCR)-amplified from genomic DNA and cloned into the XhoI and EcoRV sites of the pGL4.10-E4TATA. This vector contains a 50-bp minimal E4TATA promoter. The cloned segments ranged between $115 \mathrm{bp}$ and $195 \mathrm{bp}$ in size. Site-directed mutagenesis was used to create the altered allele for each SNP using the GeneArt Site-Directed Mutagenesis System (Thermo Fisher Scientific). All the constructs were validated by Sanger sequencing.

\section{Transfection and dual luciferase reporter assay}

For the luciferase reporter assay, TPC-1 and BCPAP cells were transiently transfected with reporter plasmids using Lipofectamine 2000 reagents (Thermo Fisher Scientific) according to the manufacturer's instructions. Briefly, cells were seeded in 24 -well plates and grown to $\sim 85 \%$ confluence at the time of transfection. Each well was transfected with 250 ng luciferase reporter plasmid and 1.25 ng Renilla plasmid pRL-TK (Promega Madison, WI) as an internal control for each well. Cells were lysed $24 \mathrm{~h}$ after transfection with $100 \mu \mathrm{l}$ passive lysis buffer (Promega) per well. A 20- $\mu$ l aliquot of cell lysate was assayed for luciferase activity using the GloMax 96 Microplate Luminometer (Promega).

\section{Chromatin immunoprecipitation assay}

Chromatin immunoprecipitation (ChIP) assays to determine the degree of SMAD3 protein enrichment were performed using the Magna ChIP A/G Chromatin Immunoprecipitation Kit (Millipore Sigma) according to the manufacturer's instructions. Briefly, TPC-1 cells were seeded in 150-mm dishes and grown to confluence before harvesting. Cells were fixed with $1 \%$ formaldehyde for $10 \mathrm{~min}$ at room temperature. After sonication, chromatin was immunoprecipitated with a rabbit anti-SMAD3 antibody (ab28379, Abcam, Cambridge, UK) or IgG (\#2729, Cell Signaling Technology, Boston, MA) at $4{ }^{\circ} \mathrm{C}$ overnight. The protein/DNA complexes were eluted from the magnetic beads after standard washing steps. The cross-links were reversed by incubating at $62{ }^{\circ} \mathrm{C}$ for $2 \mathrm{~h}$ and $95^{\circ} \mathrm{C}$ for $10 \mathrm{~min}$. Final DNA products were purified and used as templates for quantitative real-time PCR (qPCR) with primers covering the candidate SMAD3 protein binding sites in the SPRY4 upstream region.

\section{Microarray hybridization following transfection with si- SMAD3}

TPC-1 cells were seeded in a six-well plate and grown to $\sim 85 \%$ confluence at the time of transfection. 25 pmol siSMAD3 or si-Control were transfected into the cells using $4.5 \mu l$ Lipofectamine RNAiMAX (Thermo Fisher Scientific). After transfection for $24 \mathrm{~h}$, the cells were harvested and total RNA was extracted using the Trizol reagent (Thermo Fisher Scientific) then treated by DNase-I (Thermo Fisher Scientific) to eliminate DNA contamination. Experiments described above were performed at three different time points to obtain three replicates.

RNA concentration was determined using the Qubit 2.0 Fluorometer (Agilent Technologies, Santa Clara, CA) with an RNA HS Assay Kit. The integrity of the RNA samples was assessed by BioAnalyzer (Agilent Technologies). All RNA integrity numbers were greater than 9.0. Clariom D Human arrays (Thermo Fisher Scientific) targeting more than 542,500 transcripts were used to assess gene expression. Totally $100 \mathrm{ng}$ RNA was used to generate the single-stranded complementary DNA (cDNA) samples for hybridization. Then, the cDNA was enzymatically fragmented and biotinylated using the WT Terminal Labeling kit (Thermo Fisher Scientific). The cDNA 
samples were hybridized to the array at $45^{\circ} \mathrm{C}$ for $16 \mathrm{~h}$. The arrays were washed and scanned with the Affymetrix GeneChip Scanner 3000 7G system (Thermo Fisher Scientific) using Affymetrix GeneChip Command Console software.

Signal intensities were processed by the robust multiarray average method using Affymetrix Expression Console software. $^{20}$ The corresponding microarray data have been deposited in the Gene Expression Omnibus database (accession number GSE102225).

\section{Quantitative real-time PCR assay}

Quantitative real-time PCR assay was performed in three biological replicates on an ABI Prism 7900 HT Sequence Detection System (Thermo Fisher Scientific) according to the manufacturer's protocol. For RNA expression analysis, RNA was extracted by using the Trizol reagent (Thermo Fisher Scientific) then treated by DNase-I (Thermo Fisher Scientific) to eliminate DNA contamination. One microgram RNA was used for cDNA synthesis using High-Capacity cDNA Reverse Transcription Kit (Thermo Fisher Scientific). Taqman assays were carried out using Taqman probe sets for SMAD3 (Hs00969210_m1), SPRY4 (Hs01935412_s1), and GAPDH (4352665) with Taqman Fast Universal PCR Master Mix (Thermo Fisher Scientific). All the probe sets were purchased from Thermo Fisher Scientific. SPRY4-IT1, SPRY4-AS1, and all primer sets used in ChIP assay were detected by Fast SYBR Green Master Mix kit (Thermo Fisher Scientific). All qPCR primer sequences are provided in Supplementary Table S1 online.

\section{Statistical analysis}

Real-time PCR expression and luciferase assay data are represented by mean $\pm \mathrm{SD}$. The data were first assessed to ensure the normality by applying the Shapiro test. The homogeneous group variances were assessed by the Bartlett test. The data were then analyzed by applying the $t$-test. All $P$ values reported are two-sided. Gene canonical pathway analysis using the differential gene expression data caused by $S M A D 3$ knockdown was performed using Ingenuity Pathway Analysis software (Qiagen, Hilden, Germany). For the gene expression array analysis, a filtering method based on percentage of arrays above noise cutoff was applied to filter out low-expression genes. A linear model was employed to detect differentially expressed genes between conditions. To improve the estimates of variability and statistical tests for differential expression, a variance smoothing method with moderated t-statistic was employed. ${ }^{21}$ The significance level was adjusted by controlling the mean number of false positives. $^{22}$ Statistical software SAS 9.4 and R were used for analysis.

\section{RESULTS}

\section{Haplotype analysis reveals a 25.6-kb LD block in the SMAD3 gene}

The two GWAS tag SNPs, rs56062135 and rs2289261, are located in intron 1 and intron 2 of the SMAD3 gene, respectively. To find other variants that are co-inherited with the two SNPs, we first performed LD analysis in the 129-kb SMAD3 gene region using 1000 Genomes European population data (Figure 1). A $25.6-\mathrm{kb}$ LD block region spanning the

Chromosome 15, physical distance: $129 \mathrm{~kb}$

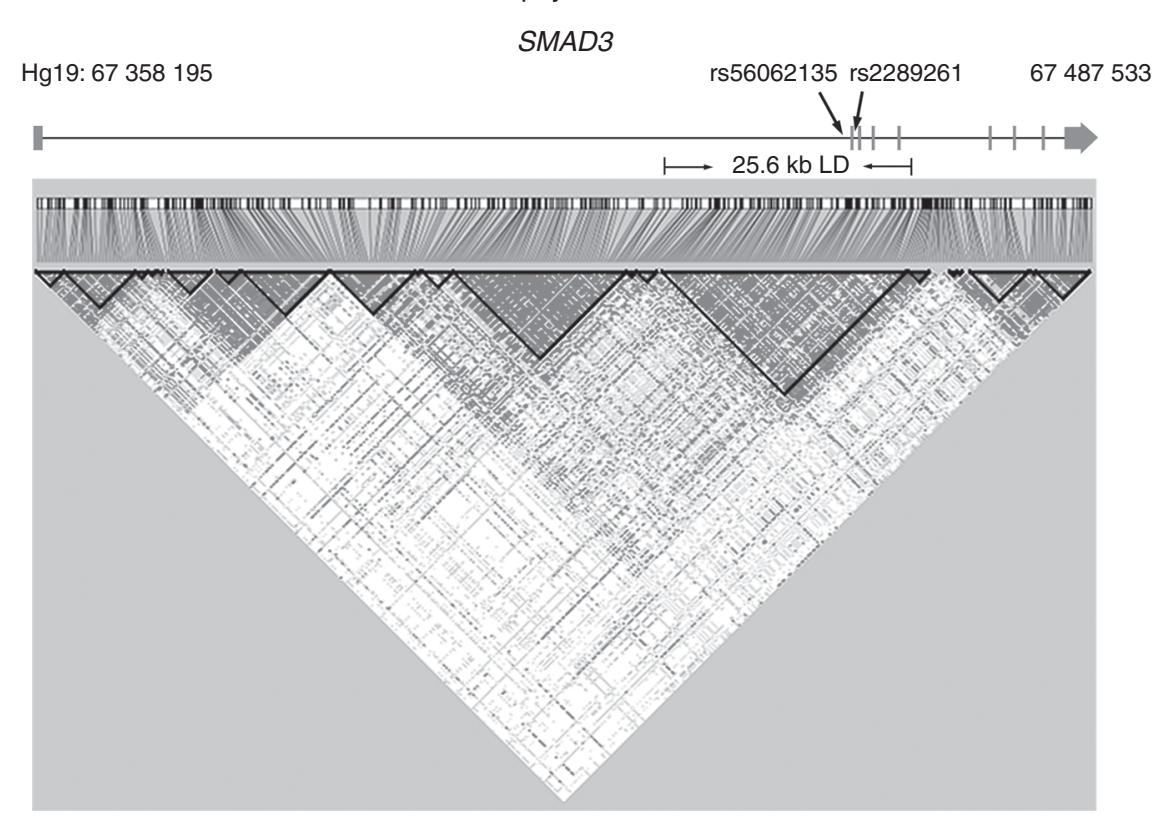

Figure 1 Linkage disequilibrium (LD) plot representation of the SMAD3 locus. The figure represents linkage disequilibrium based on 1000 Genomes European population data (phase 3) along the SMAD3 locus. The region spans chromosome 15 from 67358195 to 67487533 for a total of $129 \mathrm{~kb}$. The vertical bars represent exons and the horizontal lines represent introns. Arrows show the direction of transcription in the genome. The two genome-wide association studies (GWAS) tag single-nucleotide polymorphisms (SNPs) (rs56062135 and rs2289261) are labeled as indicated. 
chromosomal coordinates 67441506 to 67467129 (Hg19) in chromosome 15 was detected. In this block, a total of 24 SNPs, including the two tag SNPs, showed significant association with lower SMAD3 expression in noncancerous thyroid and with thyroid cancer risk (Supplementary Table S2). We hypothesize that the functional variants that can influence SMAD3 expression are embedded in the same LD region. First, to identify regulatory variants we retrieved the scores of all the 24 SNPs in the RegulomeDB database (http:// www.regulomedb.org/). These scores summarize an annotation for each SNP by predictions based on Gene Expression Omnibus, ENCODE, and other databases and assess the evidence for regulatory potential. We chose SNPs with the lowest RegulomeDB scores (scores from 1 to 3 ) plus one of the GWAS tag SNPs (rs2289261) for further study. Of the six SNPs selected, four are located in intron 1 of SMAD3.

Second, we used the six SNPs for haplotype analysis using the genotyping and imputation data from the recent GWAS. ${ }^{6}$ Three haplotypes $(P<0.01)$ showed significant differences in distribution between PTC cases and controls. Hap2 and Hap3 are PTC protective haplotypes $(\mathrm{OR}=0.69$ and 0.79 ), while Hap1 appears to be a PTC risk haplotype $(\mathrm{OR}=1.41)$. Hap1 contains the risk [C] allele of the tag SNP rs2289261, and accounts for $16 \%$ of all cases (Table 1 ).

\section{SNPs rs17293632 and rs4562997 display allele-specific enhancer activity in PTC cell lines}

To evaluate the effects of the six variants (rs1866316 T $>\mathrm{C}$, rs17293632 $\mathrm{C}>\mathrm{T}, \quad \operatorname{rs} 744910 \mathrm{~A}>\mathrm{G}, \quad \operatorname{rs8032739} \mathrm{A}>\mathrm{G}$, rs2289261 $\mathrm{C}>\mathrm{G}$, and rs4562997 $\mathrm{G}>\mathrm{A}$ ) on SMAD3 transcription, luciferase reporter assays were performed. Hypothesizing that the intronic variants play an enhancer role in the regulation of SMAD3 expression, 100-200 bp DNA fragments surrounding each SNP allele were cloned into a minimal promoter reporter vector. Luciferase activity was then measured in two PTC cell lines, TPC-1 (Figure 2a) and BCPAP (Figure 2b). Two independent clones for each of the two alleles were generated and enhancer activity was measured in quadruplicate for each group. Of the six tested variants, two SNPs (rs17293632 and rs4562997) showed significant differential enhancer activity in both cell lines $(P<0.01)$. The wild-type allele [C] of rs17293632 displayed the highest enhancer activity compared with the empty vector control in both cell lines. While the assay showed somewhat differing activity between the TPC-1 and BCPAP cell lines, no allele-specific enhancer activity was observed for rs1866316, rs744910, rs8032739, and rs2289261.

Taken together, our data demonstrate that rs17293632 and rs4562997 are functional variants that can influence transcriptional activity. We suggest that they function within cisregulatory regions in SMAD3 introns 1 and 3, respectively.

\section{Identification of differentially expressed genes caused by SMAD3 knockdown}

SMAD3 plays an important role as a direct DNA binding transcriptional factor in the TGF- $\beta$ pathway. To identify genes involved in SMAD3-mediated transcriptional regulation in thyroid, genome-wide expression analysis was carried out by using expression microarray in SMAD3 knockdown cells. TPC-1 and BCPAP cells were transiently transfected by using either SMAD3 target small interfering RNA (si-SMAD3) or scrambled small interfering RNA control (si-Control). Subsequent expression microarray analysis was conducted in the TPC-1 cell line due to its higher knockdown efficacy (Supplementary Figure S1). A total of 116 dysregulated genes were identified by comparing si-SMAD3 and si-Control treated cells in three independent experiments $(P<0.001$, fold change $>1.5)$. Of these genes, 24/116 (21\%) were upregulated (Supplementary Table S3). Biological functional assessment was performed using Ingenuity Pathway Analysis software, which showed that cancer was in the top category of "Disease and Disorders" (Supplementary Table S4). The top five categories of "Molecular and Cellular Functions" were cellular growth and proliferation, cell cycle, cellular development, cell morphology, and cellular function and maintenance (Supplementary Table S5). These data strongly implicate $S M A D 3$-regulated genes in thyroid carcinogenesis.

\section{SMAD3 acts as a repressor of SPRY4 via direct binding}

To further characterize the downstream targets of SMAD3 in thyroid, we compared the differentially expressed genes in the SMAD3 knockdown cells with the expression data of 59 tumor/normal thyroid pairs from the Cancer Genome Atlas data portal (https://tcga-data.nci.nih.gov/docs/publications/ tcga/). Only the genes that showed either upregulation or downregulation in both groups described above were included. The sprouty RTK signaling antagonist 4 (SPRY4) gene was found to be the second-most dysregulated candidate gene $\left(P=2.69 \times 10^{-11}\right)$ (Supplementary Table S6). As one of the top 25 dysregulated genes, SPRY4 was found to be upregulated while SMAD3 expression was decreased

Table 1 Haplotype analysis of the SMAD3 locus in PTC cases versus controls

\begin{tabular}{|c|c|c|c|c|c|c|c|c|c|c|}
\hline \multirow[t]{2}{*}{ Haplotype ID } & \multicolumn{2}{|c|}{$\begin{array}{c}\text { Haplotype } \\
\text { frequencies }\end{array}$} & \multirow[b]{2}{*}{ OR } & \multicolumn{7}{|c|}{ SNP ID } \\
\hline & Controls & Cases & & $P$ Value $^{b}$ & rs1866316 & rs17293632 & rs744910 & rs8032739 & rs2289261 & rs4562997 \\
\hline Нар \#1 & 0.12 & 0.16 & 1.41 & 4.64E-06 & $C$ & $\mathrm{~T}$ & G & G & $C$ & A \\
\hline Нар \#3 & 0.11 & 0.09 & 0.79 & 7.02E-03 & T & C & G & $A$ & $\mathrm{G}$ & G \\
\hline
\end{tabular}

OR, odds ratio; PTC, papillary thyroid carcinoma; SNP, single-nucleotide polymorphism.

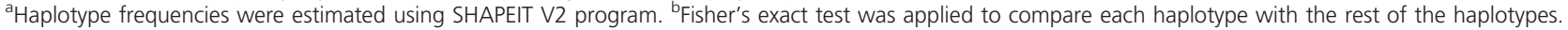



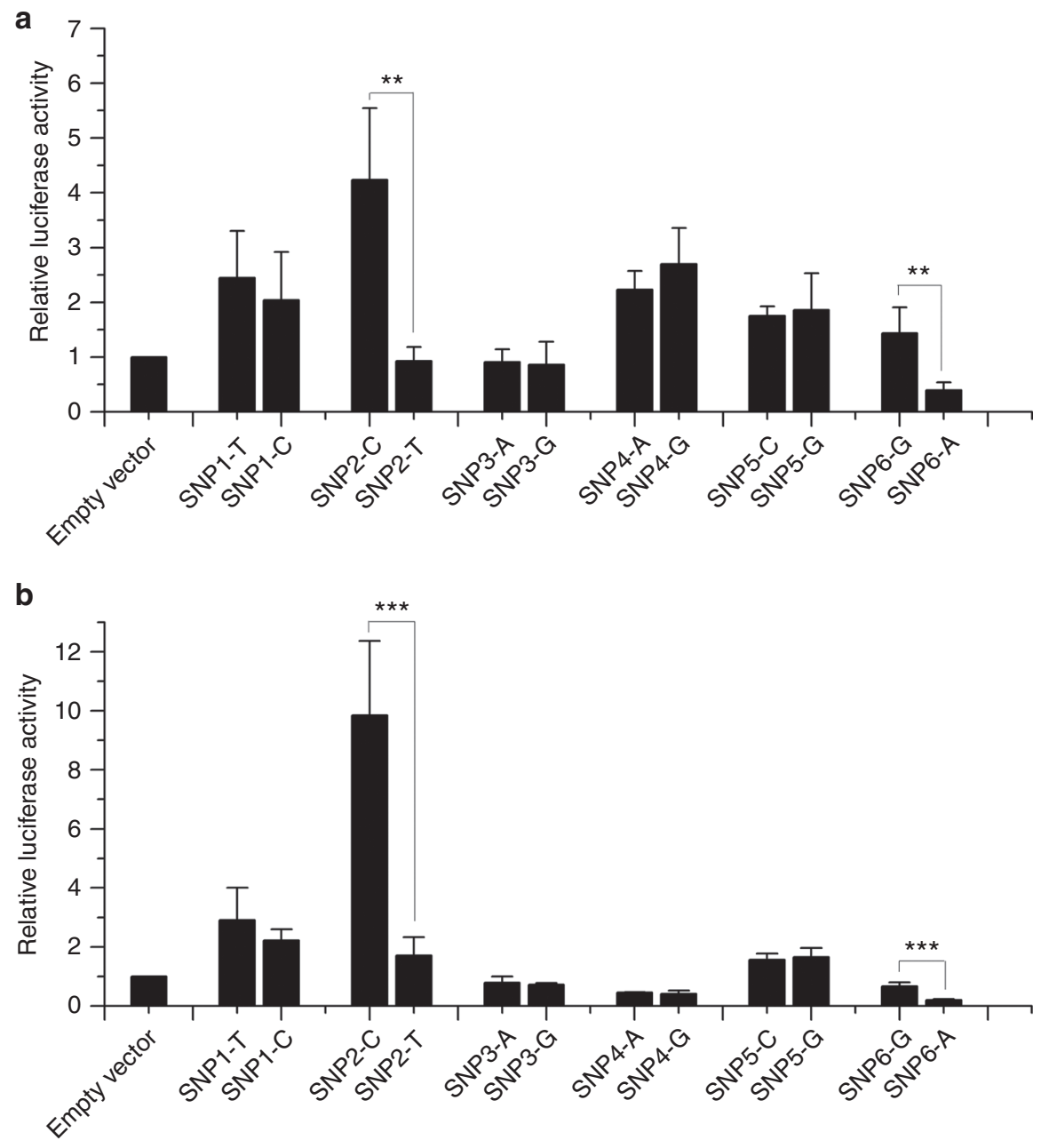

Figure 2 Transcriptional activity of the candidate SMAD3 intronic variants with different alleles. Dual reporter luciferase assay using pGL4.10E4TATA constructs with either the wild-type or risk allele of the six selected single-nucleotide polymorphisms (SNPS) in (a) TPC-1 cells and (b) BCPAP cells. Firefly luciferase values were normalized to cotransfected Renilla values. All values were normalized with the values of the corresponding groups transfected with empty vector. Results are shown as means \pm SD of four independent experiments. SNP1: rs1866316; SNP2: rs17293632; SNP3: rs744910; SNP4: rs8032739; SNP5: rs2289261; SNP6: rs4562997. For each SNP, the left allele is the major allele. Each group was repeated four times. $* * P<0.01 ; * * * P<0.001$. Two-tailed $t$-test, $n=4$.

(Figure 3a). SPRY4 is located in 5q31 (chromosome 5, genomic coordinates 141689992 to 141704620 ). Two long noncoding RNAs in this locus, SPRY4-IT1 (SPRY4 intronic transcript 1) and SPRY4-AS1 (SPRY4 antisense 1), were transcribed in opposite orientation. To validate the expression change of SPRY4, along with the two long noncoding RNAs, qPCR assays were performed in SMAD3 knockdown PTC cell lines (Figures 3b,c). While both SPRY4 and SPRY4-IT1 showed significantly differential expression in the TPC-1 cell line, no significant difference was observed for SPRY4-AS1 (Figure 3d). This implies that SMAD3 functions as a transcriptional repressor of SPRY4 and SPRY4-IT1, but not SPRY4-AS1.

In an attempt to understand the role of SMAD3 as a SPRY4 repressor via binding, we searched the $15-\mathrm{kb}$ upstream region of SPRY4 for potential SMAD3 protein binding sites. SMAD binding elements (SBEs) have been defined as a palindromic sequence including $5^{\prime}$ GTCT-3' and its complement, 5'AGAC-3' (Figure 4a). Many SMAD-responsive regions include more than one SBE, which usually contains extra bases between the palindromic sequence. ${ }^{14,23} \mathrm{~A}$ total of five potential SBEs were found upstream of SPRY4 with less than 5 bp between the two palindromic sequences (Figure $4 \mathbf{b}$ ). To validate the existence of the SBEs, subsequent ChIP assays using a SMAD3 antibody were conducted in TPC-1 cells. Significant DNA enrichment was observed in all five sites (Figure 4c).

Taken together, SMAD3 is a SPRY4 inhibitor via direct binding at multiple sites in the regulatory region upstream of SPRY4.

\section{DISCUSSION}

Thyroid cancer is a complex disease that has one of the strongest genetic components of all cancers. ${ }^{24}$ To identify the 


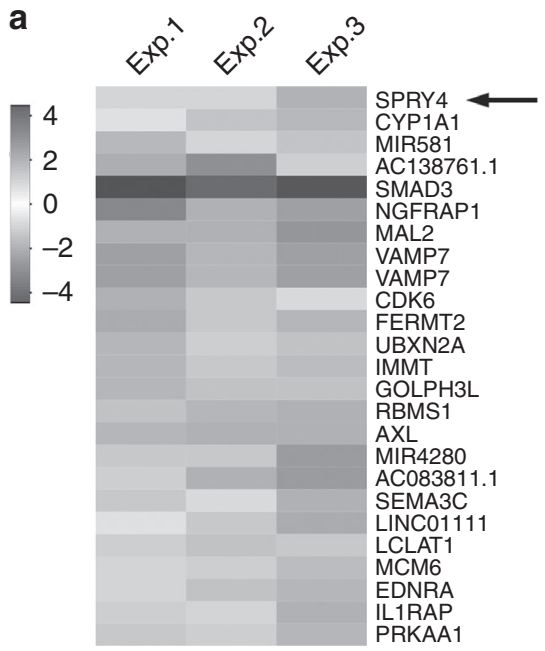

b
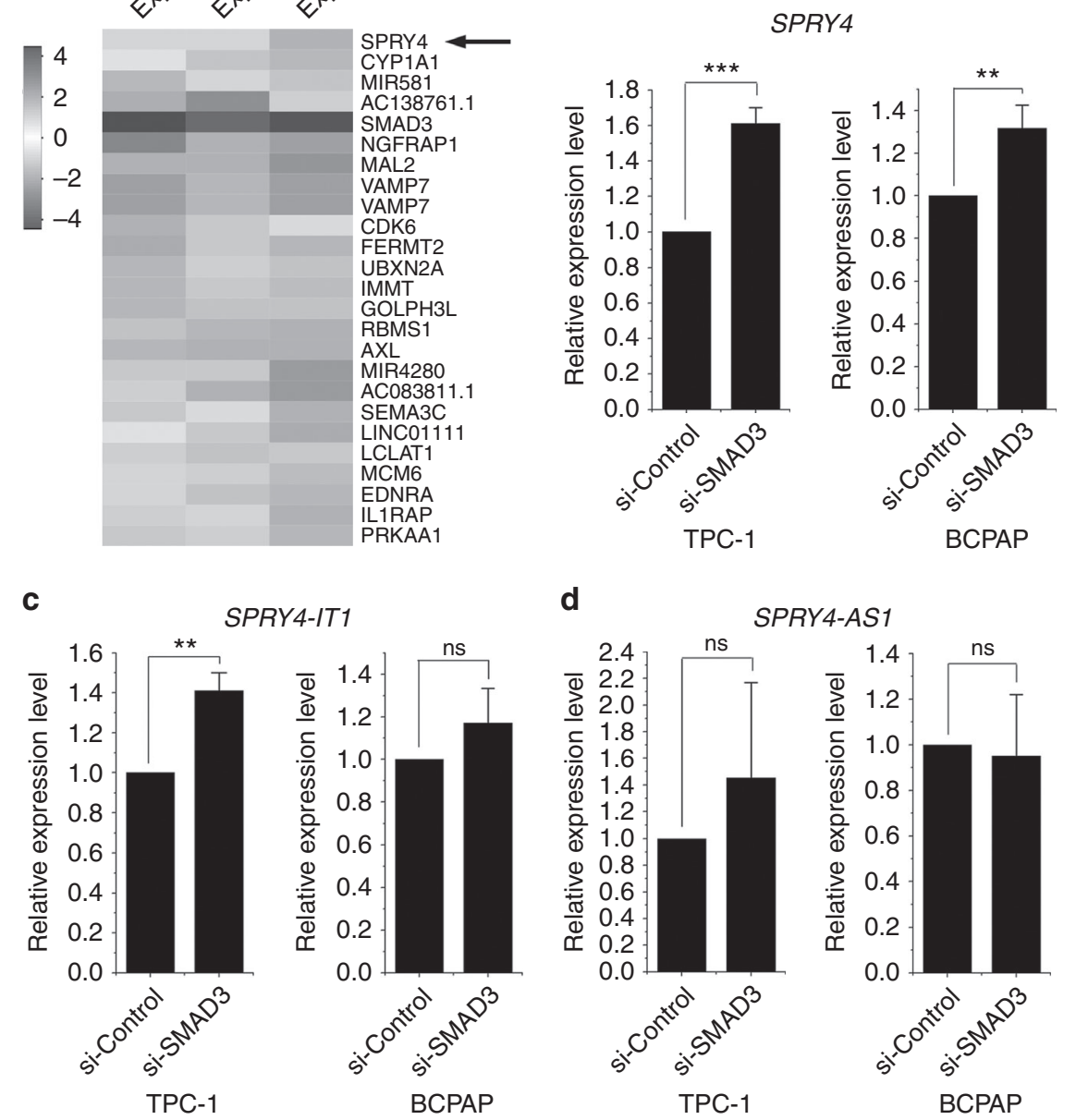

SPRY4-IT1

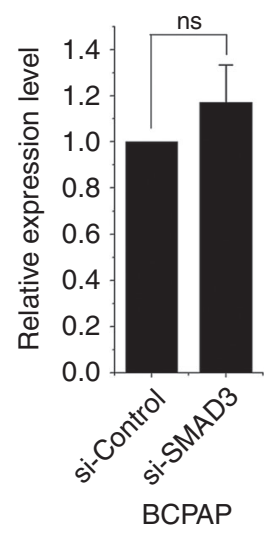

d

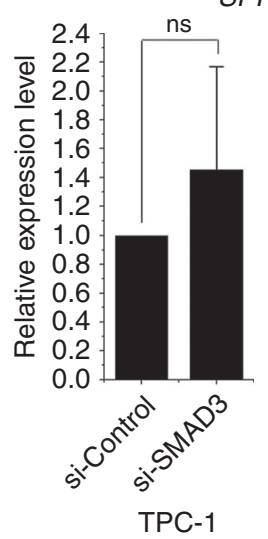

SPRY4-AS1

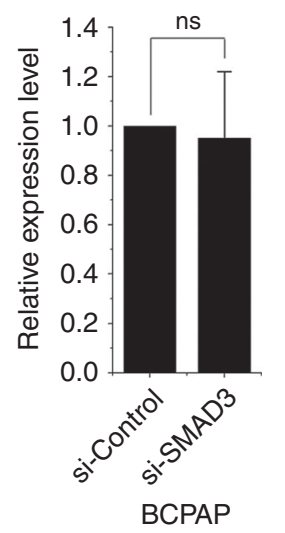

Figure 3 Gene expression profile of SMAD3 knockdown in papillary thyroid carcinoma (PTC) cell lines. (a) Gene expression differences of the top 25 genes dysregulated by SMAD3 knockdown in TPC-1 cell lines. The expression is plotted with heat-map color scale using relative expression fold change (si-SMAD3 treated cells versus si-control treated cells) (fold change $>1.5, P<0.001, n=3$ ). Quantitative real-time polymerase chain reaction (qPCR) validations for (b) SPRY4, (c) SPRY4-IT1, and (d) SPRY4-AS1 in TPC-1 and BCPAP cell lines.

low-penetrance genes and risk loci that lead to thyroid cancer, several GWAS have been performed in distinct populations. ${ }^{3,6,25,26}$ A number of genetic variants have been reported to be involved in thyroid cancer predisposition. Some of them, such as the risk loci at 9q22 and 14q13, have been well characterized by elucidating the relevant molecular mechanism in thyroid tumorigenesis. ${ }^{4,5}$ A recent GWAS identified five novel thyroid cancer risk loci. In the present work, we provide functional annotations for one of these loci located at 15q22 and containing the coding gene SMAD3.

$S M A D 3$ encodes a key regulator in the TGF- $\beta$ pathway that activates or represses target gene transcription. Variants in protein binding hot-spots on SMAD3 can reduce the binding to its interacting proteins and cause a range of quantitative changes in the expression of genes induced by SMAD3. ${ }^{27}$ For instance, typical $S M A D 3$ coding variants can lead to increased aortic expression of several key players in the TGF- $\beta$ pathway and cause a syndromic form of aortic aneurysms and dissections with early-onset osteoarthritis. ${ }^{28,29}$
Here, we identified two SMAD3 intronic variants (rs17293632 and rs4562997) that can cause allele-specific dysregulation as determined by luciferase assays. We propose that the intronic regions where the two variants are located are likely to be enhancer elements. Previous reports have found that intron-containing genes show higher transcriptional levels when compared with intronless genes. ${ }^{30}$ Regulatory elements such as enhancers are often located at a distance from the target gene but can form a complex communication with the promoter region. However, recent studies suggest that some elements located within introns can also work in combination and collaboration with promoters. ${ }^{31,32}$ Our results support this hypothesis and provide another example of regulatory intronic variants in a coding gene. In addition, we demonstrate how the germ-line genotypes of these variants affect the genetic susceptibility to thyroid cancer.

SPRY4 is a member of the sprouty family, which is recognized as a key regulator of the ERK signaling 
a

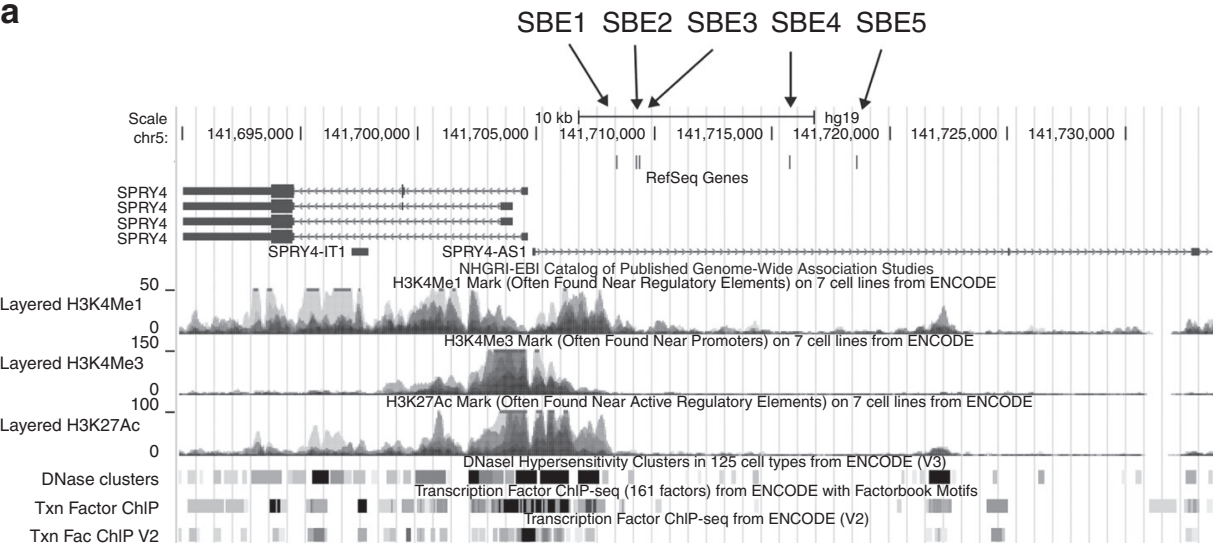

b

\begin{tabular}{|c|c|c|}
\hline Locus & Genomic coordinates & Potential binding sequence \\
\hline SBE1 & chr5:141708 296-141708 306 & AGACTCCGTCT \\
\hline SBE2 & chr5:141708 $139-141709147$ & $\underline{\text { GTCTCAGAC }}$ \\
\hline SBE3 & chr5:141708 $342-141709351$ & $\underline{\text { AGACCAGTCT }}$ \\
\hline SBE4 & chr5:141715787-141715808 & AGACCATAT $\underline{\text { GTCTAAGATAGAC }}$ \\
\hline SBE5 & chr5:141718 $535-141718545$ & $\underline{\text { AGACCTT }}$ \\
\hline
\end{tabular}

C

SPRY4-SBE1

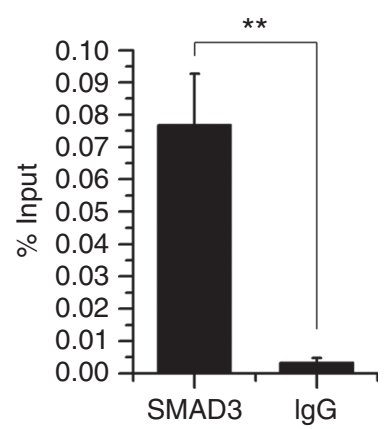

SPRY4-SBE2

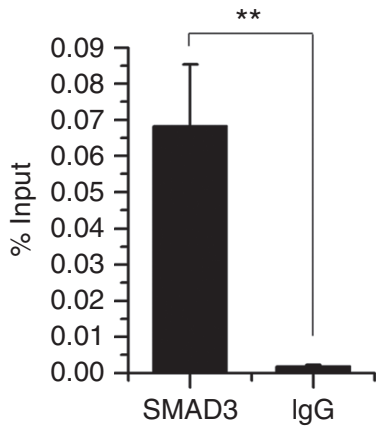

SPRY4-SBE3

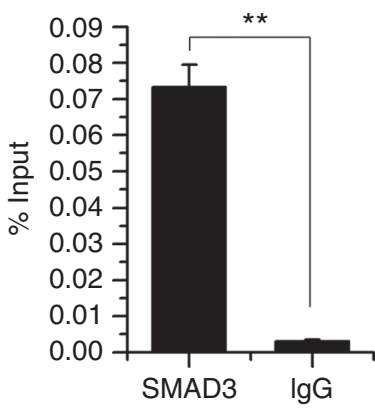

SPRY4-SBE4

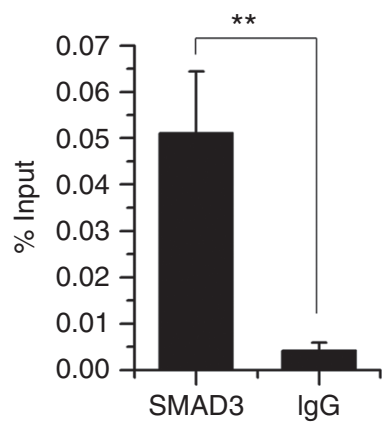

SPRY4-SBE5

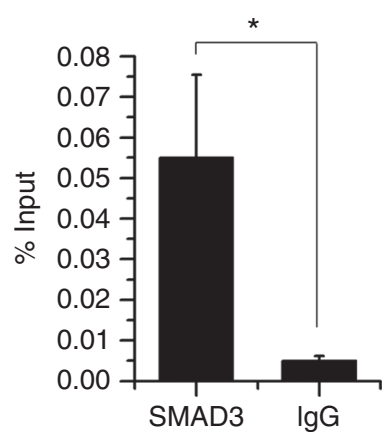

Figure 4 SMAD3 binding sites in the upstream region of SPRY4. (a) Genomic annotation of the SPRY4 gene and $15 \mathrm{~kb}$ of its upstream region in the University of California-Santa Cruz Genome Browser (Hg19). Chromatin regulatory features such as DNase hypersensitivity sites, histone modifications, and predicted transcription factor binding from chromatin immunoprecipitation (ChIP)-seq data are included. The locations of the five SMAD binding elements (SBEs) are labeled as indicated. (b) The corresponding genomic coordinates and key genomic features of each SBE of SPRY4. The potential binding sequences are underlined. (c) ChIP assays for the five SBEs in TPC-1 cells. Values represent the percentage of the corresponding input DNA control. Results were shown as means \pm SD of three replicates. Each group was repeated three times. ${ }^{*} P<0.05 ; * \star P<0.01$. 
pathway. ${ }^{33}$ It has been reported that SPRY4 can inhibit cell proliferation, migration, and invasion in non-small cell cancer and breast cancer cell lines. ${ }^{34,35}$ Downregulation of SPRY4 in matched prostate normal and tumor tissue pairs was also reported. ${ }^{36}$ Our results show that SPRY4 is downregulated by $S M A D 3$ via direct binding at five $\mathrm{SBE}$ sites. Moreover, we found that its intronic long noncoding RNA, SPRY4-IT1, is also regulated by SMAD3. SPRY4-IT1 has been described to be involved in metastasis, apoptosis, and proliferation in several cancer types. ${ }^{37,38}$ Our data suggest a role for SMAD3 as a repressor at this locus, but the detailed regulatory mechanism is still unclear. It remains to be elucidated whether additional hitherto unrecognized proteins act with SMAD3 in modulating expression. Based on their important cellular functions, we speculate that the dysregulation of these genes, notably SMAD3 and its targets SPRY4 and SPRY4-IT1, will impact the typical cellular signaling network and promote uncontrolled cell proliferation and metastasis in thyroid cancer.

There are two highly conserved domains in SMAD proteins, $\mathrm{MH} 1$ and $\mathrm{MH} 2$. The MH1 domain is necessary for sequencespecific DNA binding and transcription. While some GC-rich DNA fragments have been discovered to have a binding affinity with SMAD proteins, ${ }^{39}$ the $\mathrm{MH} 1$ domain mainly recognizes the featured sequences that form a SBE palindrome. ${ }^{40}$ Here, we validated the existence of multiple SBEs in the region upstream of the SPRY4 gene. Our results show that the SBE can function as a SMAD3 binding site with more than one nucleotide between the two palindromic sequences. It not only broadens the definition of SBE, but also further proves the correlation between SMAD3 and the two upregulated genes, SPRY4 and SPRY4-IT1.

In conclusion, our study provides a functional characterization of the genomic variants that have a causal role in creating thyroid cancer risk. The expression of SMAD3 in thyroid cells is regulated by enhancers located in introns of the gene. The enhancers in turn are regulated in an allelespecific manner by the functional genomic variants. We also validate the regulatory mechanism for SPRY4 and SPRY4-IT1 by SMAD3 protein as a transcriptional factor. Our data support an important role of SMAD3, a key member of TGF$\beta$ signaling, in thyroid cancer predisposition.

\section{SUPPLEMENTARY MATERIAL}

Supplementary material is linked to the online version of the paper at http://www.nature.com/gim

\section{ACKNOWLEDGMENTS}

This work was supported by National Cancer Institute grants P30CA16058 and P50CA168505. We thank Matthew D. Ringel for helpful discussions and suggestions. We also thank the Ohio State University Comprehensive Cancer Center Genomics Shared Resource for help with microarray hybridization. The analysis was done in part by an allocation of computing time from the Ohio Supercomputer Center.

\section{AUTHOR CONTRIBUTIONS}

Y.W., H.H., and A.d.I.C. designed the experiments; Y.W. and L.G. performed the experiments; S.L. performed LD and haplotype analysis; L.Y. performed expression analysis for microarray assays; W.L., J.P., R.S., and P.B. contributed reagents and tissue samples; Y.W., A.d.I.C., and H.H. wrote the paper.

\section{DISCLOSURE}

The authors declare no conflict of interest.

\section{REFERENCES}

1. Nagy $R$, Ringel MD. Genetic predisposition for nonmedullary thyroid cancer. Horm Cancer. 2015;6:13-20.

2. Fagin JA, Wells Jr. SA, Biologic and clinical perspectives on thyroid cancer. N Engl J Med. 2016;375:2307.

3. Gudmundsson J, Sulem P, Gudbjartsson DF, et al. Common variants on $9 q 22.33$ and $14 q 13.3$ predispose to thyroid cancer in European populations. Nat Genet. 2009;41:460-464.

4. Wang $\mathrm{Y}, \mathrm{He} \mathrm{H}$, Li W, et al. MYH9 binds to IncRNA gene PTCSC2 and regulates FOXE1 in the 9q22 thyroid cancer risk locus. Proc Natl Acad Sci USA. 2017:114:474-479.

5. Jendrzejewski J, He HL, Radomska HS, et al. The polymorphism rs944289 predisposes to papillary thyroid carcinoma through a large intergenic noncoding RNA gene of tumor suppressor type. Proc Natl Acad Sci USA. 2012;109:8646-8651.

6. Gudmundsson J, Thorleifsson G, Sigurdsson JK, et al. A genome-wide association study yields five novel thyroid cancer risk loci. Nat Commun. 2017:8:14517.

7. Yanagisawa K, Osada H, Masuda A, et al. Induction of apoptosis by Smad3 and down-regulation of Smad3 expression in response to TGF-beta in human normal lung epithelial cells. Oncogene. 1998;17:1743-1747.

8. Xue J, Lin X, Chiu WT, et al. Sustained activation of SMAD3/SMAD4 by FOXM1 promotes TGF-beta-dependent cancer metastasis. I Clin Invest. 2014;124:564-579.

9. Tang PM, Zhou S, Meng XM, et al. Smad3 promotes cancer progression by inhibiting E4BP4-mediated NK cell development. Nat Commun. 2017;8:14677.

10. Han SU, Kim HT, Seong DH, et al. Loss of the Smad3 expression increases susceptibility to tumorigenicity in human gastric cancer. Oncogene. 2004;23:1333-1341.

11. Wolfraim LA, Fernandez TM, Mamura M, et al. Loss of Smad3 in acute T-cell lymphoblastic leukemia. N Engl J Med. 2004;351:552-559.

12. Fleming $\mathrm{NI}$, Jorissen RN, Mouradov D, et al. SMAD2, SMAD3 and SMAD4 mutations in colorectal cancer. Cancer Res. 2013;73:725-735.

13. Ku JL, Park SH, Yoon KA, et al. Genetic alterations of the TGF-beta signaling pathway in colorectal cancer cell lines: a novel mutation in Smad3 associated with the inactivation of TGF-beta-induced transcriptional activation. Cancer Lett. 2007;247:283-292.

14. Massague J, Seoane J, Wotton D. Smad transcription factors. Genes Dev. 2005; 19:2783-2810.

15. Millet C, Zhang YE. Roles of Smad3 in TGF-beta signaling during carcinogenesis. Crit Rev Eukaryot Gene Expr. 2007;17:281-293.

16. Zhang $Y$, Handley D, Kaplan $T$, et al. High throughput determination of TGFbeta1/SMAD3 targets in A549 lung epithelial cells. PLoS One. 2011;6:e20319.

17. Mullen AC, Orlando DA, Newman JJ, et al. Master transcription factors determine cell-type-specific responses to TGF-beta signaling. Cell. 2011;147:565-576.

18. Barrett JC, Fry B, Maller J, Daly MJ. Haploview: analysis and visualization of LD and haplotype maps. Bioinformatics. 2005;21:263-265.

19. Delaneau O, Marchini J, Zagury JF. A linear complexity phasing method for thousands of genomes. Nat Methods. 2011;9:179-181.

20. Irizarry RA, Hobbs B, Collin F, et al. Exploration, normalization, and summaries of high density oligonucleotide array probe level data. Biostatistics. 2003;4:249-264.

21. Yu L, Gulati P, Fernandez S, Pennell M, Kirschner L, Jarjoura D. Fully moderated T-statistic for small sample size gene expression arrays. Stat Appl Genet Mol Biol. 2011;10:42.

22. Gordon A, Glazko G, Qiu X, Yakovlev A. Control of the mean number of false discoveries, Bonferroni and stability of multiple testing. Ann Appl Stat. 2007;1:179-190. 
23. Tone $Y$, Furuuchi K, Kojima Y, Tykocinski ML, Greene MI, Tone M. Smad3 and NFAT cooperate to induce Foxp3 expression through its enhancer. Nat Immunol. 2008;9:194-202.

24. Goldgar DE, Easton DF, Cannonalbright LA, Skolnick MH. Systematic population-based assessment of cancer risk in first-degree relatives of cancer probands. J Natl Cancer Inst. 1994;86:1600-1608.

25. Gudmundsson J, Sulem P, Gudbjartsson DF, et al. Discovery of common variants associated with low TSH levels and thyroid cancer risk. Nat Genet. 2012;44:319-322.

26. Son HY, Hwangbo Y, Yoo SK, et al. Genome-wide association and expression quantitative trait loci studies identify multiple susceptibility loci for thyroid cancer. Nat Commun. 2017;8:15966.

27. Schiro MM, Stauber SE, Peterson TL, et al. Mutations in protein-binding hotspots on the hub protein Smad3 differentially affect its protein interactions and Smad3-regulated gene expression. PLoS One. 2011;6:e25021.

28. van de Laar IM, Oldenburg RA, Pals G, et al. Mutations in SMAD3 cause a syndromic form of aortic aneurysms and dissections with early-onset osteoarthritis. Nat Genet. 2011;43:121-126.

29. Regalado ES, Guo DC, Villamizar C, et al. Exome sequencing identifies SMAD3 mutations as a cause of familial thoracic aortic aneurysm and dissection with intracranial and other arterial aneurysms. Circ Res. 2011;109:680-U220.

30. Shabalina SA, Ogurtsov AY, Spiridonov AN, Novichkov PS, Spiridonov NA, Koonin EV. Distinct patterns of expression and evolution of intronless and intron-containing mammalian genes. Mol Biol Evol. 2010;27:1745-1749.

31. Stadhouders $R$, van den Heuvel A, Kolovos $P$, et al. Transcription regulation by distal enhancers: who's in the loop? Transcription. 2012;3:181-186.
32. Ott CJ, Blackledge NP, Kerschner JL, et al. Intronic enhancers coordinate epithelial-specific looping of the active CFTR locus. Proc Natl Acad Sci USA. 2009;106:19934-19939.

33. Masoumi-Moghaddam S, Amini A, Morris DL. The developing story of Sprouty and cancer. Cancer Metastasis Rev. 2014;33:695-720.

34. Vanas V, Muhlbacher E, Kral R, Sutterluty-Fall H. Sprouty4 interferes with cell proliferation and migration of breast cancer-derived cell lines. Tumour Biol. 2014;35:4447-4456.

35. Tennis MA, Van Scoyk MM, Freeman SV, Vandervest KM, Nemenoff RA, Winn RA. Sprouty-4 inhibits transformed cell growth, migration and invasion, and epithelial-mesenchymal transition, and is regulated by Wnt7A through PPARgamma in non-small cell lung cancer. Mol Cancer Res. 2010;8:833-843.

36. Wang JH, Thompson B, Ren CX, Ittmann M, Kwabi-Addo B. Sprouty4, a suppressor of tumor cell motility, is downregulated by DNA methylation in human prostate cancer. Prostate. 2006;66:613-624.

37. Shen F, Cai WS, Feng Z, et al. Long non-coding RNA SPRY4-IT1 promotes colorectal cancer metastasis by regulate epithelial-mesenchymal transition. Oncotarget. 2017;8:14479-14486.

38. Mazar J, Zhao W, Khalil AM, et al. The functional characterization of long noncoding RNA SPRY4-IT1 in human melanoma cells. Oncotarget. 2014;5:8959-8969.

39. Baburajendran N, Jauch $R$, Tan CYZ, Narasimhan K, Kolatkar PR. Structural basis for the cooperative DNA recognition by Smad4 MH1 dimers. Nucleic Acids Res. 2011;39:8213-8222.

40. Malhotra N, Kang J. SMAD regulatory networks construct a balanced immune system. Immunology. 2013;139:1-10. 